\title{
FUNCTIONS WITH STRICTLY CONVEX EPIGRAPH
}

\author{
StéPHANe Simon ANd PATRICK Verovic
}

Abstract. The aim of this paper is to provide a full and simple characterization of functions with domain in an arbitrary topological real vector space whose epigraph is strictly convex. In order to achieve this description, we first give both affine and topological properties of strictly convex subsets of general topological real vector spaces. Then, we investigate the relationship between functions and their epigraph from the geometric and topological viewpoints.

Mathematics subject classification (2010): Primary: 52A07, Secondary: 52A05.

Keywords and phrases: Convexity, affine geometry, strict convexity, topological vector spaces.

\section{REFERENCES}

[1] L. P. Belluce, W. A. Kirk AND E. F. Steiner, Normal structure in Banach spaces, Pac. J. Math. 26, 3 (1968), 433-440.

[2] N. BourbaKI, Espaces vectoriels topologiques - Chapitres 1 à 5, Springer, 1981.

[3] N. L. CAROTHERS, A short course on Banach space theory, Cambridge University Press, 2004.

[4] I. Ekeland AND R. Temam, Convex analysis and variational problems, Society for Industrial and Applied Mathematics, 1999.

[5] N. Hadjis AVVAS, S. Komlosi AND S. Schaible, Handbook of generalized convexity and generalized monotonicity Springer, 2005.

[6] W. B. Johnson AND J. Lindenstrauss, Handbook of the geometry of Banach spaces. Volume 1, North-Holland, 2001.

[7] J. L. Kelley and I. Namioka, Linear topological spaces, D. Van Nostrand Company, 1963.

[8] J. Lindenstrauss, On nonseparable reflexive Banach spaces, Bull. Am. Math. Soc. 72, 6 (1966), 967-970.

[9] R. T. Rockafellar, Convex analysis, Princeton University Press, 1970.

[10] R. Webster, Convexity, Oxford University Press, 1994. 